\title{
Effect of conductive layer condition on the morphology of anodic aluminum oxide template-assisted indium antimonide nanowires
}

\begin{abstract}
Indium antimonide nanowires were synthesized by electrochemical deposition using anodic aluminum oxide template in the presence of gold film as conductive layers. Field emission scanning electron microscopy and energy dispersive X-ray spectrometry measurements were carried out to investigate the effect of adhesive insulated tape covered below the conductive layer. Results showed that the anodic aluminum oxide template covered with insulating tapes had better morphology with less presence of overgrown rough film on the topside of the anodic aluminum oxide template and it exhibited a smoother nanowire sidewall as compared to the uncovered ones. Additionally, the unique properties of anodic aluminum oxide were controllable pore diameter with a narrow size distribution at some intervals. It was evident from the energy dispersive $\mathrm{X}$-ray spectrum that the nanowires synthesized from the covered template condition exhibited better InSb composition and stoichiometric ratio compared to the uncovered template condition.
\end{abstract}

Keyword: AAO template; Conductive layer; Electrodeposition; InSb Nanowires; Insulating tape 
